\title{
FUNCTIONAL ITERATION AND THE JOSEPHUS PROBLEM
}

\author{
by ANDREW M. ODLYZKO and HERBERT S. WILF $\dagger$
}

(Received 27 February, 1990)

1. Introduction. The problem of Josephus is the following. We are given two positive integers $n, q$. There are $n$ places arranged around a circle, and numbered clockwise $1,2, \ldots, n$. Each of $n$ people takes one of the places, then (please excuse this, but we didn't invent the problem!) every $q$ th one is executed, until just one remains. More precisely, the occupant of place $q$ is 'removed' first, and in general, if some place $j$ has just been vacated, then the $q$ th one of the places clockwise around from $j$ that are still occupied will be vacated next. One question is this: if you would like to be the last survivor, then into what place should you go initially? We denote the answer to this question by $J_{q}(n)$. For example, if $n=5$ and $q=2$, the order of execution is $2,4,1,5,3$, and $J_{2}(5)=3$. Other questions have been raised about the problem, and it has an extensive literature (see [1]-[10]). In this paper we deal with the $J_{q}(n)$ 's.

Here we observe that in one of the known algorithms for solving the problem, the sequence of numbers that is generated is remarkably well approximated by a single term of its asymptotic series. This result, which essentially is a property of the iterated 'ceiling' function, is both of independent interest and also permits us to find an explicit-looking formula for $J_{3}(n)((5)$ below).

For a fixed $\alpha>1$ we study the sequence $f_{0}=1, f_{n+1}=\left\lceil\alpha f_{n}\right\rceil(n \geq 0)$ (we use ' $\lceil\cdot\rceil$ ' and ' $\lfloor\cdot J$ ' for the ceiling and the floor functions, respectively). We show that although these iterates grow exponentially fast, they are approximable to within $O(1)$ by a single term of their asymptotic expansions.

2. Results. In [3, Section 3.3], an interesting approach to the Josephus problem is described, and the authors give the following procedure for finding $J_{q}(n)$.

(a) Define a sequence $\left\{D_{k}^{(a)}\right\}_{k \geq 0}$ by

$$
D_{k}^{(q)}=\left\lceil\frac{q}{q-1} D_{k-1}^{(q)}\right\rceil \quad\left(k \geq 1 ; D_{0}^{(q)}=1\right) .
$$

(b) Determine the least integer $k$ such that $D_{k}^{(q)}>(q-1) n$.

(c) Then the answer is $J_{q}(n)=q n+1-D_{k}^{(q)}$.

We study the behavior of the $D_{k}^{(q)}$. The striking feature that we find is that they are extremely well approximated by the first term of their asymptotic formulas, for large $k$ and fixed $q$.

THEOREM 1. For each integer $q \geq 2$ there is a real number $K(q)$ such that

$$
D_{k}^{(q)}=K(q)\left(\frac{q}{q-1}\right)^{k}+\epsilon_{k . q},
$$

in which $\epsilon_{k, 2}=0$ for all $k$ and if $q \geq 3$ then

$$
-(q-2)<\epsilon_{k, q} \leq 0 \quad(k \geq 0) .
$$

† Research supported by the U.S. Office of Naval Research.

Glasgow Math. J. 33 (1991) 235-240. 
As a trivial consequence, note that since $K(2)=1$, the well known formula

$$
J_{2}(n)=1+2\left(n-2^{\left\lfloor\log _{2} n\right\rfloor}\right) \quad(n=0,1, \ldots)
$$

holds.

Corollary 1. We have the 'exact formula'

$$
D_{k}^{(3)}=\left\lfloor K(3)\left(\frac{3}{2}\right)^{k}\right\rfloor \quad(k=0,1, \ldots),
$$

and so we have also the 'exact formula'

$$
J_{3}(n)=3 n+1-\left\lfloor K(3)\left(\frac{3}{2}\right)^{\left[\log _{32}((2 n+1) / K(3))\right]}\right\rfloor \quad(n=0,1, \ldots),
$$

where $K(3)=1.62227050288476731595695098289932411 \ldots$. .

3. Proofs. We will prove a little more than is necessary for Theorem 1 above. Fix $\alpha>1$, and let $f(x)=\lceil\alpha x\rceil$. We study the iterates $f_{n}=f_{n}(\alpha)$ of $f$, defined by

$$
f_{n+1}=f\left(f_{n}\right)=\left\lceil\alpha f_{n}\right\rceil \quad\left(n \geq 0 ; f_{0}=1\right) .
$$

Proposition 1. There exists a constant $c=c(\alpha)$ such that

Proof. Define

$$
f_{n}(\alpha) \sim c(\alpha) \alpha^{n} \quad(n \rightarrow \infty) .
$$

$$
u_{n}=f_{n} / \alpha^{n} .
$$

We claim that $\left\{u_{n}\right\}$ is increasing and bounded from above. It increases because

$$
\alpha^{n+1} u_{n+1}=\left\lceil\alpha^{n+1} u_{n}\right\rceil \geq \alpha^{n+1} u_{n},
$$

and is bounded from above because

implies that

$$
\alpha^{n+1} u_{n+1}=\left\lceil\alpha^{n+1} u_{n}\right\rceil \leq 1+\alpha^{n+1} u_{n}
$$

$$
u_{n+1} \leq u_{n}+\alpha^{-n-1} \quad(n \geq 0)
$$

which in turn implies that $u_{n} \leq \alpha /(\alpha-1)$ for all $n \geq 0$.

We now study the error term in the asymptotic formula (7). The next proposition shows that the error is very small in many cases.

Proposition 2. If $\alpha \geq 2$ or if $\alpha=2-1 / m$ for integer $m \geq 2$, then

$$
\forall n \geq 0: f_{n}=\left\lfloor c(\alpha) \alpha^{n}\right\rfloor \text {. }
$$

Proof. We define numbers $\left\{e_{n}\right\}$ by

$$
f_{n}=\alpha f_{n-1}+e_{n}=\left\lceil\alpha f_{n-1}\right\rceil \quad(n \geq 1),
$$

so $0 \leq e_{n}<1$. With the $u_{n}$ of (8) above, we have

$$
f_{n}=u_{n} \alpha^{n}=\alpha u_{n-1} \alpha^{n-1}+e_{n}=u_{n-1} \alpha^{n}+e_{n},
$$

from which $u_{n}=u_{n-1}+e_{n} / \alpha^{n}$ and

$$
u_{n}=1+\sum_{k=1}^{n} \frac{e_{k}}{\alpha^{k}} \rightarrow c(\alpha)=1+\sum_{k=1}^{\infty} \frac{e_{k}}{\alpha^{k}} .
$$


It follows that

and that

$$
f_{n}=c(\alpha) \alpha^{n}-\sum_{j \geq 1} \frac{e_{n+j}}{\alpha^{j}}
$$

$$
\left|f_{n}-c(\alpha) \alpha^{n}\right|<\sum_{j \geq 1} \frac{1}{\alpha^{j}}=\frac{1}{\alpha-1} .
$$

Thus $0<c(\alpha) \alpha^{n}-f_{n}<1 /(\alpha-1)$ for all $n$, and the result follows.

Finally, if $\alpha$ is rational we can bound the $e_{n}$ 's away from 1 and extend the result slightly. Indeed, suppose $\alpha=2-1 / m$ for integer $m \geq 2$. Then (9) shows that all $\left|e_{n}\right| \leq(m-1) / m$, and $(10)$ yields

$$
\left|f_{n}-c(\alpha) \alpha^{n}\right| \leq \frac{m-1}{m} \cdot \frac{1}{\alpha-1}=1 .
$$

However it cannot happen that all $e_{n}=(m-1) / m$ for $n>n_{0}$, for otherwise we would have $f_{n}=c(\alpha)(2-1 / m)^{n}-1$, but the right side cannot be an integer for all $n>n_{0}$, completing the proof.

To finish the proof of Theorem 1 we return to the parameter values that occur in the Josephus problem. Let $\alpha=q /(q-1)$ and write $K(q)$ for $c(\alpha)$ in (5), to find that

Now from (9),

$$
D_{k}^{(q)}=K(q)\left(\frac{q}{q-1}\right)^{k}-\sum_{j \geq 1} e_{k+j}\left(\frac{q-1}{q}\right)^{k} .
$$

$$
e_{k}=\left\lceil\left(\frac{q}{q-1}\right) D_{k-1}^{(q)}\right\rceil-\left(\frac{q}{q-1}\right) D_{k-1}^{(q)}
$$

and so $(q-1) e_{k}$ is an integer in the range $[-(q-2), 0]$. The estimate (3) of Theorem 1 now follows, and the proof is complete.

4. The function $c(\alpha)$. In this section we study the 'constant' $c(\alpha)$, as a function of $\alpha$. A brief table of $c(\alpha)$, showing some of its irregular behavior, is in Table 1.

A graph of $c(\alpha)$, for $1.1<\alpha<2.5$ is shown in Figure 1 .

TABle 1. Some values of $c(\alpha)$

\begin{tabular}{cc}
\hline$\alpha$ & $c(\alpha)$ \\
\hline $1 \cdot 050000$ & $11 \cdot 8354164 \ldots$ \\
$1 \cdot 125000$ & $5 \cdot 1954489 \ldots$ \\
$1 \cdot 1250+$ & $5 \cdot 8448800 \ldots$ \\
$1 \cdot 166666$ & $4 \cdot 0371021 \ldots$ \\
$1 \cdot 1666+$ & $4 \cdot 7099524 \ldots$ \\
1.200000 & $3 \cdot 2438724 \ldots$ \\
$1.2000+$ & $3 \cdot 8926469 \ldots$ \\
1.250000 & $2 \cdot 0763957 \ldots$ \\
1.500000 & $1 \cdot 6222705 \ldots$ \\
1.900000 & $1 \cdot 2701620 \ldots$ \\
$2 \cdot 000000$ & $1 \cdot 0000000 \ldots$ \\
$2 \cdot 0000+$ & $2 \cdot 0000000 \ldots$ \\
2.500000 & $1 \cdot 3653870 \ldots$ \\
5.500000 & $1 \cdot 1311946 \ldots$ \\
\hline
\end{tabular}




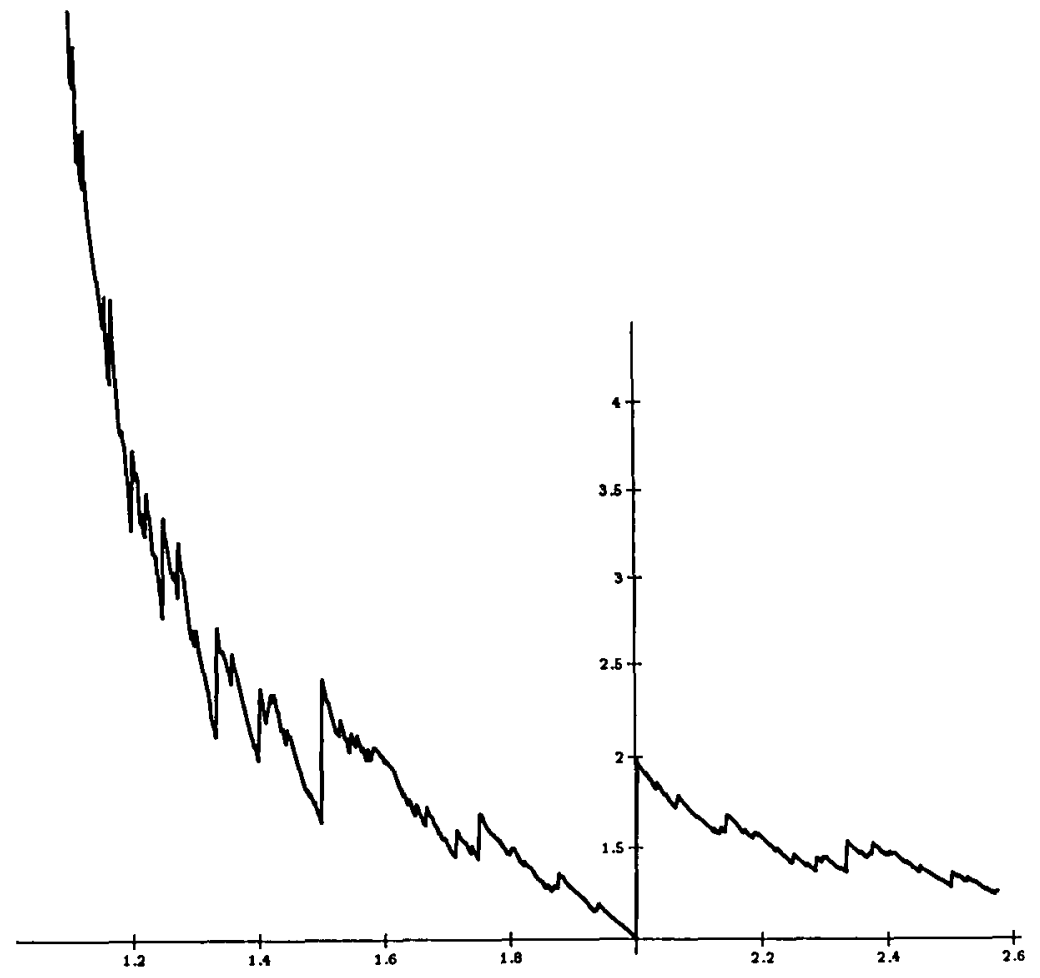

Figure 1. $c(\alpha)$ vs. $\alpha$.

It is easy to see that at the integers the function $c(\alpha)$ has jump discontinuities of the following kind:

$$
c(m+)=c(m)+\frac{1}{m-1}=1+\frac{1}{m-1} \quad(m=2,3, \ldots) .
$$

We are also able to make a quantitative statement about the jumps at the Josephus points, as the following proposition shows.

Proposition 3. At the Josephus points $\alpha_{q}=q /(q-1)$, the function $c(\alpha)$ has jump discontinuities of the form

$$
c(\alpha+)=\alpha c(\alpha) \quad(\alpha=2,3 / 2,4 / 3,5 / 4, \ldots) .
$$

Proof. We claim that at such a value of $\alpha$ the sequences $\left\{f_{n}(\alpha)\right\}$ and $\left\{f_{n}(\alpha+)\right\}$ are related by

$$
f_{n}(\alpha)=f_{n-1}(\alpha+)+1 \quad(n \geq 1) .
$$

which would establish the truth of the proposition. To prove (12), it suffices to show that $\left\{f_{n}(\alpha)-1\right\}$ satisfies the same recurrence that $\left\{f_{n-1}(\alpha+)\right\}$ does, namely the recurrence

$$
\left\lceil(\alpha+\epsilon)\left(f_{n}(\alpha)-1\right)\right\rceil=f_{n+1}(\alpha)-1 \text {, }
$$

for each $n \geq 1$ and all small enough $\epsilon$. 
But the left side is

$$
\begin{aligned}
\left\lceil\alpha f_{n}(\alpha)-\alpha+\epsilon f_{n}(\alpha)-\epsilon\right\rceil & =\left\lceil\alpha f_{n}(\alpha)-1+(1-\alpha-\epsilon)+\epsilon f_{n}(\alpha)\right\rceil \\
& =\left\lceil\alpha f_{n}(\alpha)+\epsilon f_{n}(\alpha)-\left(\epsilon+\frac{1}{q-1}\right)\right]-1 \\
& =\left\lceil\frac{q}{q-1} f_{n}(\alpha)+\epsilon f_{n}(\alpha)-\left(\epsilon+\frac{1}{q-1}\right)\right]-1
\end{aligned}
$$

If $(q-1) \mid f_{n}(\alpha)$ then the last member above is $(q /(q-1)) f_{n}(\alpha)-1$, i.e., it is $f_{n+1}(\alpha)-1$, as required.

Next suppose that $f_{n}(\alpha)=(q-1) m+r(1 \leqslant r \leqslant q-2)$. Then the last member above is

$$
\begin{aligned}
\left\lceil q m+\frac{q r}{q-1}+\epsilon\left(f_{n}(\alpha)\right.\right. & \left.-1)-\frac{1}{q-1}\right\rceil-1 \\
& =q m+r+\left\lceil\frac{r-1}{q-1}+\epsilon\left(f_{n}(\alpha)-1\right)\right]-1=q m+r=f_{n+1}(\alpha)-1 .
\end{aligned}
$$

5. Remarks, and a conjecture. (i) As it stands, our 'explicit' formula for $J_{3}(n)$ is not a dramatic improvement over the algorithm in (1), because the computation of the universal constant $K(3)$ requires the $D_{k}$ 's of (1). This situation could change if some independent method were found to calculate $K(3)$ with high precision.

(ii) We would like to know more about the function $c(\alpha)$. In particular, does it satisfy some functional equation? Can one evaluate it at the Josephus points in some way that is quite independent of the algorithm (2)? What more can be proved about the sizes of its discontinuities?

(iii) If $q>2$ then in view of the uncertainty (3) in the formula (2), what we know is that $D_{k}^{(q)}$ is one of the $q-2$ numbers

$$
\left\lfloor K(q)\left(\frac{q}{q-1}\right)^{k}\right\rfloor-i \quad(i=0, \ldots, q-3) .
$$

This means that the correct value of $J_{n}(q)$ is known to be one of $q-2$ consecutive places around the circle (the chance of survival has increased from $1: n$ to $1:(q-2)$ ).

(iv) We have a conjecture about the error in the general asymptotic formulas above. In the Josephus case, where $\alpha=q /(q-1)$, we conjecture that the integers $(q-1) e_{n}$, which assume only the values $0,1, \ldots, q-2$, in fact are asymptotically uniformly distributed on those $q-1$ values, and furthermore that small sets of successive values are asymptotically independent. This would imply that if $q \geq 2$,

$$
\lim _{k \rightarrow \infty} \operatorname{Prob}\left\{D_{k}^{(q)}-\left\{K(q)\left(\frac{q}{q-1}\right)^{k}-\frac{(q-2)}{2}\right\} \leq t \sqrt{\frac{q(q-2)}{12(2 q-1)}}\right\}=\frac{1}{\sqrt{2 \pi}} \int_{-\infty}^{t} e^{-y^{2 / 2}} d y .
$$

This conjecture is likely to be quite difficult to settle. 


\section{REFERENCES}

1. W. E. M. G. Ahrens, Mathematische Unterhaltungen und Spiele, (Teubner, 1918) 118-169.

2. D. J. Aulicino, and $M$. Goldfield, A new relation between primitive roots and permutations, Amer. Math. Monthly 76 (1969), 664-666.

3. R. L. Graham, D. E. Knuth and O. Patashnik, Concrete Mathematics, (Addison-Wesley, 1989).

4. I. N. Herstein and I. Kaplansky, Matters mathematical (Chelsea, New York, 1978) $121-128$.

5. F. Jakobczyk, On the generalized Josephus problem, Glasgow Math. J. 14 (1973), $168-173$.

6. D. E. Knuth, The Art of Computer Programming, Vol. 1 (Addison-Wesley, 1973), Ex. 1.3.2.22, and Vol. III (1975), Ex. 5.1.1.2. $262-270$.

7. E. L. Lloyd, An $O(n \log m)$ algorithm for the Josephus problem, J. Algorithms 4 (1983),

8. R. A. MacFhraing, (R. A. Rankin), Aireamh muinntir Fhinn is Dhubhain, agus sgeul Iosephuis is an dà thichead ludhaich (The numbering of Fionn's and Dubhan's men, and the story of Josephus and the forty Jews), Proc. Royal Irish Acad. Sect. A 52 (1948), 87-93 (Gaelic. English summary; MR 10 (1949), 509).

9. W. J. Robinson, The Josephus problem, Math. Gaz. 44 (1960), 47-52. 207-218.

10. D. Woodhouse, The extended Josephus problem, Rev. Mat. Hisp.-Amer. (Ser. 4) 31 (1973),

AT \& T BELl LABORATORIES

MuRray Hill

NJ 07974

USA
University of Pennsylvania

Philadelphia, PA 19104-6395

USA 